\title{
Operator representation of the Dirac Coulomb Green function and relativistic polarizability of hydrogen-like atoms
}

\author{
Le Anh Thu†, Le Van Hoang $\dagger$, L I Komarov $\dagger$ and $T$ S Romanova \\ † Department of Theoretical Physics, Belarussian State University, 4 Fr. Skariny av., \\ Minsk 220050, Republic of Belarus \\ $\ddagger$ Institute of Physico-Chemical Problems, Belarussian State University, 14 Leningradskaya str., \\ Minsk 220080, Republic of Belarus \\ $\S$ Institute of Nuclear Problems, Belarussian State University, 14 Leningradskaya str., \\ Minsk 220080, Republic of Belarus
}

Received 24 February 1994, in final form 29 June 1994

\begin{abstract}
The Dirac Coulomb Green function in the representation of the annihilation and creation operators is established and on the basis of the application of this new operator representation an algebraic method of calculation of atomic characteristics is briefly discussed. The relativistic polarizability of hydrogen-like atoms is calculated and its simple analytical formula is obtained.
\end{abstract}

\section{Introduction}

The coordinate representation of the Coulomb Green function for the Dirac equation was first established by Martin and Glauber (1958) and has been used in the calculation of various problems in atomic and nuclear physics (see, for example, Zapryagaev et al 1985 and references therein). In concrete calculations some other different representations of this function are often used: the impulse representation (Gorshkov 1964, Manakov et al 1973), the coordinate representation in the expansion in power series of the Laguerre polynomial (Zon et al 1972, Zapryagaev and Manakov 1981)... However, the use of all the above-mentioned representations always leads to an enormous number of calculations of multi-dimensional integrals of special functions. This circumstance strongly decreases the efficiency of the application of analytical methods in perturbation theory with the use of the Dirac Coulomb Green function (in many papers this function is called the relativistic Coulomb Green function, see Swainson and Drake (1991)).

Currently the tendency to use the connection between the four-dimensional harmonic oscillator problem and the problem of a hydrogen atom in electromagnetic fields is intensively developing (see, for example, Komarov and Romanova 1982, Kibler and Negadi 1983, Le Van Hoang et al 1989). For relativistic Coulomb problems, a similar treatment was proposed in Komarov and Romanova (1985) and Le Van Hoang et al (1992) where, instead of the Dirac equation, an application was demonstrated of the equation in twodimensional complex space ( $\xi$-space), the set of solutions of which contains the solutions of the Dirac equation. The structure of the equation in $\xi$-space is simple in the sense that the equation term describing the interaction can be presented in the polynomial form of the coordinates and, therefore, is convenient for use in concrete algebraic calculations. In the 
present paper, on the basis of the equation suggested in Komarov and Romanova (1985), we establish the relativistic Coulomb Green function in the representation of the annihilation and creation operators; moreover this representation is established in such a way that all operators can be transformed into the normal form thereby allowing the use of the algebraic method of calculation. The use of an algebraic treatment does not need the explicit form of the wavefunctions, which enables us to reduce rather complicated calculations of the matrix elements with the Coulomb wavefunctions to a purely algebraic procedure of transforming the products of the creation and annihilation operators to a normal form (see Le Van Hoang et al 1989).

As a specific example of the application of the operator representation of the relativistic Coulomb Green function, we consider the problem of calculation of the static relativistic polarizability of hydrogen-like atoms, which has been the subject of many papers (Bartlett and Power 1969, Zon et al 1972, Labzowsky 1973, Barut and Nagel 1976, McDowell and Porter $1977, \ldots$ ) and, in our view, is still of great interest for further research. We give in detail an analysis of and obtain a simple analytical formula for the relativistic polarizability in the ground state of hydrogen-like atoms with nuclear charge ranging from 1 to 137.

\section{Equation in two-dimensional complex space}

Let us consider the following equation:

$$
\begin{aligned}
& \hat{H} \Psi(\xi)=Z e^{2} \Psi(\xi) \\
& \hat{H}=-\frac{1}{2} \mathrm{i} \alpha_{\lambda}\left(\tau_{\lambda}\right)_{s t}\left(\xi_{t} \frac{\partial}{\partial \xi_{s}}+\xi_{s}^{*} \frac{\partial}{\partial \xi_{t}^{*}}\right)+(m \beta-\varepsilon) \xi_{s} \xi_{s}^{*}
\end{aligned}
$$

where the four-component spinor $\Psi(\xi)$ is a function of the complex coordinates $\xi_{s}(s=$ $1,2) ; \alpha_{\lambda}(\lambda=1,2,3)$ and $\beta$ are Dirac matrices. Henceforth, we use the usual representation

$$
\Psi=\left(\begin{array}{l}
\Psi_{1} \\
\Psi_{2}
\end{array}\right) \quad \alpha_{\lambda}=\left(\begin{array}{cc}
0 & \sigma_{\lambda} \\
\sigma_{\lambda} & 0
\end{array}\right) \quad \beta=\left(\begin{array}{cc}
1 & 0 \\
0 & -1
\end{array}\right)
$$

where $\Psi_{1}$ and $\Psi_{2}$ are two-component spinors; $\sigma_{\lambda}(\lambda=1,2,3)$ are Pauli matrices. In the Hamiltonian (2) $\tau_{\lambda}(\lambda=1,2,3)$ are Pauli matrices operating in the space of coordinates $\xi$, which are regarded as spinor components.

As shown in Komarov and Romanova (1985), after transformation into the usual threedimensional space with the use of the correlations

$$
x_{\lambda}=\xi_{s}^{*}\left(\tau_{\lambda}\right)_{s t} \xi_{t} \quad \chi=\arg \left(\xi_{1}\right)
$$

equations (1) and (2) (for the class of wavefunctions, independent of the variable $\chi$ ) lead to the Dirac equation for a charged particle with mass $m$ and energy $\varepsilon$, moving in the Coulomb field $-Z e^{2} / r$. The existence of the above-mentioned connection allows us to use (1) and (2) in considering the relativistic Coulomb problems. For the problem of the motion of a Dirac particle in the Coulomb field plus an electromagnetic field $(A, \varphi)$ we can use the following more generalized equation in $\xi$-space:

$\left\{-\frac{1}{2} \mathrm{i} \alpha_{\lambda}\left(\tau_{\lambda}\right)_{s t}\left(\xi_{t} \frac{\partial}{\partial \xi_{s}}+\xi_{s}^{*} \frac{\partial}{\partial \xi_{t}^{*}}\right)+\left(e \alpha_{\lambda} A_{\lambda}+m \beta+\varphi-\varepsilon\right) \xi_{s} \xi_{s}^{*}-Z e^{2}\right\} \Psi(\xi)=0$

(see Le Van Hoang et al 1992). The condition of independence of the wavefunctions from the variable $x$

$$
-i \frac{\partial}{\partial \chi} \Psi(\xi)=0
$$


can be rewritten as follows as an equation in $\xi$-space:

$$
\hat{Q} \Psi(\xi)=0
$$

where

$$
\hat{Q}=\xi_{s} \frac{\partial}{\partial \xi_{s}}-\xi_{s}^{*} \frac{\partial}{\partial \xi_{s}^{*}} .
$$

In concrete calculations, one usually needs equations for two-component spinors $\Psi_{1}, \Psi_{2}$ (quadratic Dirac equations). Therefore, we now establish the corresponding equations in $\xi$-space. By making the substitutions

$$
\Psi_{1}=\sqrt{m+\varepsilon}(\tilde{F}+\widetilde{G}) \quad \Psi_{2}=\mathrm{i} \sqrt{m-\varepsilon}\left(\xi_{u} \xi_{u}^{*}\right)^{-1} \sigma_{\lambda}\left(\tau_{\lambda}\right)_{s t} \xi_{s}^{*} \xi_{t}(\tilde{F}-\widetilde{G})
$$

we transform (1) and (2), respectively, into the forms

$$
\begin{aligned}
& \left(\xi_{s} \frac{\partial}{\partial \xi_{s}}+\xi_{s}^{*} \frac{\partial}{\partial \xi_{s}^{*}}+\omega \xi_{s} \xi_{s}^{*}-\frac{Z e^{2} \varepsilon}{\omega}\right) \widetilde{F}-\left(1+\sigma_{\lambda} \hat{l}_{\lambda}+\frac{Z e^{2}}{\omega}\right) \widetilde{G}=0 \\
& -\left(1+\sigma_{\lambda} \hat{l}_{\lambda}-\frac{Z e^{2}}{\omega}\right) \widetilde{F}+\left(\xi_{s} \frac{\partial}{\partial \xi_{s}}+\xi_{s}^{*} \frac{\partial}{\partial \xi_{s}^{*}}-\omega \xi_{s} \xi_{s}^{*}+\frac{Z e^{2} \varepsilon}{\omega}\right) \widetilde{G}=0
\end{aligned}
$$

where $\omega=\sqrt{m^{2}-\varepsilon^{2}}$ (henceforth, we consider the electron mass $m=1$ ); $\hat{l}_{\lambda}$ is the orbital momentum operator, which in $\xi$-space has the form

$$
\hat{l}_{\lambda}=-\frac{1}{2}\left(\tau_{\lambda}\right)_{s t}\left(\xi_{t} \frac{\partial}{\partial \xi_{s}}-\xi_{s}^{*} \frac{\partial}{\partial \xi_{t}^{*}}\right) .
$$

The fact that the operator $\hat{\kappa}=1+\sigma_{\lambda} \hat{l}_{\lambda}$ commutes with all operators occurring in (7) and (8) permits us to exclude one of the wavefunctions $\widetilde{F}$ and $\widetilde{G}$ from the equations, and as a result, obtain the following equations for each wavefunction:

$$
\begin{aligned}
& \left\{\left(\xi_{s} \frac{\partial}{\partial \xi_{s}}+\xi_{s}^{*} \frac{\partial}{\partial \xi_{s}^{*}}\right)^{2}-\left(\omega \xi_{s} \xi_{s}^{*}\right)^{2}+\left(2 Z e^{2} \varepsilon+\omega\right) \xi_{s} \xi_{s}^{*}-\hat{\kappa}^{2}+Z^{2} e^{4}\right\} \tilde{F}=0 \\
& \left\{\left(\xi_{s} \frac{\partial}{\partial \xi_{s}}+\xi_{s}^{*} \frac{\partial}{\partial \xi_{s}^{*}}\right)^{2}-\left(\omega \xi_{s} \xi_{s}^{*}\right)^{2}+\left(2 Z e^{2} \varepsilon-\omega\right) \xi_{s} \xi_{s}^{*}-\hat{\kappa}^{2}+Z^{2} e^{4}\right\} \tilde{G}=0 .
\end{aligned}
$$

Equations (10) and (11) can be considered as having the quadratic form in $\xi$-space for the Dirac Coulomb particle. The scalar product of wavefunctions in $\xi$-space is defined by the following correlation:

$$
\langle\tilde{\Psi} \mid \tilde{\varphi}\rangle=\int \mathrm{d}^{4} \xi \widetilde{\Psi}^{*}\left(\xi_{1}^{\prime}, \xi_{1}^{\prime \prime}, \xi_{2}^{\prime}, \xi_{2}^{\prime \prime}\right) \widetilde{\varphi}\left(\xi_{1}^{\prime}, \xi_{1}^{\prime \prime}, \xi_{2}^{\prime}, \xi_{2}^{\prime \prime}\right)
$$

where $\xi_{s}^{\prime} \equiv \operatorname{Re} \xi_{s}, \xi_{s}^{\prime \prime} \equiv \operatorname{Im} \xi_{s}$.

From (1) and (2) and (10) and (11) it is easy to see that the term which describes interaction in these equations is presented in a polynomial coordinate form. This circumstance allows us (in the next section) to establish algebraic solutions for the Coulomb problem, which serve as a convenient basis of wavefunctions for effective use in different calculations, in particular, for establishing a new operator representation of the relativistic Coulomb Green function. 


\section{Algebraic solutions of the Dirac equation for the Coulomb problem}

The algebraic method for solving the Dirac equation for a charged particle in the Coulomb field was proposed by Komarov and Romanova (1985) on the basis of the application of (1) and (2). In this section, for further use, we develop this method and give the angular part of wavefunctions in general form.

Let us define operators

$$
\begin{array}{ll}
a_{s}=\sqrt{\frac{\omega}{2}}\left(\xi_{s}+\frac{1}{\omega} \frac{\partial}{\partial \xi_{s}^{*}}\right) & b_{s}=\sqrt{\frac{\omega}{2}}\left(\xi_{s}^{*}+\frac{1}{\omega} \frac{\partial}{\partial \xi_{s}}\right) \\
a_{s}^{+}=\sqrt{\frac{\omega}{2}}\left(\xi_{s}^{*}-\frac{1}{\omega} \frac{\partial}{\partial \xi_{s}}\right) & b_{s}^{+}=\sqrt{\frac{\omega}{2}}\left(\xi_{s}-\frac{1}{\omega} \frac{\partial}{\partial \xi_{s}^{*}}\right)
\end{array}
$$

where the parameter $\omega$ was defined in section 2 . Operators (13) satisfy the commutation correlations

$$
\left[a_{s}, a_{t}^{+}\right]=\delta_{s t} \quad\left[b_{s}, b_{t}^{+}\right]=\delta_{s t}
$$

(we have written only non-zero commutators). The possibility of using the algebraic method is conditioned by the fact that, all 'physical' operators can be expessed through the following 15 operators:

$$
\begin{array}{ll}
M=a_{s} b_{s} & M^{+}=a_{s}^{+} b_{s}^{+} \quad N=a_{s}^{+} a_{s}+b_{s}^{+} b_{s} \\
m_{\lambda}=\left(\tau_{\lambda}\right)_{s t} a_{t} b_{s} \quad & m_{\lambda}^{+}=\left(\tau_{\lambda}\right)_{s t} a_{s}^{+} b_{t}^{+} \\
n_{\lambda}^{a}=\left(\tau_{\lambda}\right)_{s t} a_{s}^{+} a_{t} \quad n_{\lambda}^{b}=\left(\tau_{\lambda}\right)_{s t} b_{t}^{+} b_{s}
\end{array}
$$

which form a closed algebra $S O(4,2)$ simply isomophic to the algebra of the group of dynamical symmetry of the system described by (1) and (2) (see Komarov and Romanova 1985). For example, in the representation (13)-(15) the operators occurring in (7), (8) and (10), (11) have the form

$\xi_{s} \frac{\partial}{\partial \xi_{s}}+\xi_{s}^{*} \frac{\partial}{\partial \xi_{s}^{*}}=M-M^{+}-2 \quad \xi_{s} \xi_{s}^{*}=\frac{1}{2 \omega}\left(M+M^{+}+N+2\right)$

$\hat{l}_{\lambda}=\frac{1}{2}\left(n_{\lambda}^{a}-n_{\lambda}^{b}\right) \quad x_{\lambda}=\left(\tau_{\lambda}\right)_{s t} \xi_{s}^{*} \xi_{t}=\frac{1}{2 \omega}\left(m_{\lambda}^{+}+m_{\lambda}+n_{\lambda}^{a}+n_{\lambda}^{b}\right)$.

Let us now establish a basis of wavefunction vectors, which belong to a given value of the square of the orbital momentum $l$ and a given value of its third projection $m$. Naturally, the basis wavefunction vectors have to belong to the zero eigenvalue of the operator $\hat{Q}=a_{s}^{+} a_{s}-b_{s}^{+} b_{s}$, i.e. they should be established through the vectors

$$
\left(a_{1}^{+}\right)^{n_{1}}\left(a_{2}^{+}\right)^{n_{3}}\left(b_{1}^{+}\right)^{m_{1}}\left(b_{2}^{+}\right)^{m_{2}}|0(\omega)\rangle
$$

where positive integers $n_{1}, n_{2}, m_{1}, m_{2}$ satisfy the correlation

$$
n_{1}+n_{2}=m_{1}+m_{2} \text {. }
$$

$|0(\omega)\rangle$ is a vacuum state, defined by the equations

$$
\left.a_{s} \mid 0(\omega)\right\}=b_{s}|0(\omega)\rangle=0 .
$$

The operator of the third projection of orbital momentum $\hat{l}_{3}$ in the representation of operators (13)-(15) has the form

$$
\hat{l}_{3}=\frac{1}{2}\left(a_{1}^{+} a_{1}-a_{2}^{+} a_{2}-b_{1}^{+} b_{1}+b_{2}^{+} b_{2}\right)
$$

therefore, it is obvious to obtain the correlation

$$
n_{1}-n_{2}-m_{1}+m_{2}=2 m
$$


where $m$ is a magnetic quantum number $(m=0, \pm 1, \pm 2, \ldots)$. Finally, taking into account the correlations (18) and (20), we find the wavefunction vector, which belongs to the given values of $l$ and $m$ in the form

$$
|l m\rangle=\left(a_{1}^{+} b_{2}^{+}\right)^{|m|} \sum_{k=0}^{N} C_{k}\left(a_{1}^{+} b_{1}^{+}\right)^{k}\left(a_{2}^{+} b_{2}^{+}\right)^{N-k}|0(\omega)\rangle
$$

for $m>0$ (for the case $m<0$ the term $a_{1}^{+} b_{2}^{+}$should be replaced by $a_{2}^{+} b_{1}^{+}$). Substituting (21) into the equation

$$
\hat{L}^{2}|l m\rangle=l(l+1)|l m\rangle
$$

with the operator of the square of the orbital momentum has the form

$$
\hat{L}^{2}=\frac{1}{2} N(N+2)-M^{+} M
$$

and by using correlations (14) and (19), we obtain

$$
\left.|l m\rangle=\left(M^{+}\right)^{J} D_{m}^{l}\left(m^{+}\right) \mid 0(\omega)\right\}
$$

where

$$
\begin{aligned}
D_{m}^{l}\left(m^{+}\right)|0(\omega)\rangle & =N_{l m}\left(a_{1}^{+} b_{2}^{+}\right)^{\mid m !} \sum_{k=0}^{l-|m|} \frac{(-1)^{k}(l+|m|+k) !}{k !(k+|m|) !(l-|m|-k) !}\left(a_{1}^{+} b_{1}^{+}\right)^{k} \\
& \times\left(M^{+}\right)^{l-|m|-k}|0(\omega)\rangle
\end{aligned}
$$

and $N_{l m}$ is a normalization constant. The existence of the operator $\left(M^{+}\right)^{j}$ in (22) is conditioned by the correlation

$$
\left[\hat{L}^{2}, M^{+}\right]=0
$$

and is needed for constructing the radiation part of wavefunctions. The eigenvectors of operator $\hat{\kappa}$ can be established in the usual way from eigenvectors $\chi^{\uparrow(\downarrow)}$ of operator $\sigma_{3}$ and from vectors $D_{m}^{l}\left(m^{+}\right)|0(\omega)\rangle$. For further use, we establish some solutions $Z_{l k}$,

$$
\begin{aligned}
& Z_{1,2}=\frac{1}{\sqrt{3}}\left(m_{3}^{+}|0\rangle \chi^{\uparrow}-\frac{1}{2}\left(m_{1}^{+}+\mathrm{i} m_{2}^{+}\right)|0\rangle \chi^{\downarrow}\right) \\
& Z_{1,-1}=\frac{1}{\sqrt{6}}\left(m_{3}^{+}|0\rangle \chi^{\uparrow}+\left(m_{1}^{+}+\mathrm{i} m_{2}^{+}\right)|0\rangle \chi^{\downarrow}\right) .
\end{aligned}
$$

In section 4 we will use the basis vectors

$$
\left(M^{+}\right)^{n} Z_{l x}\left(m^{+}\right)|0\rangle
$$

for establishing wavefunctions and, therefore, it is neccessary to take note now of some correlations with respect to these basis vectors

$$
\begin{aligned}
& M\left(M^{+}\right)^{n} Z_{l k}\left(m^{+}\right)|0\rangle=n(n+2 l+1)\left(M^{+}\right)^{n-1} Z_{l k}\left(m^{+}\right)|0\rangle \\
& N\left(M^{+}\right)^{n} Z_{l k}\left(m^{+}\right)|0\rangle=2(n+l)\left(M^{+}\right)^{n} Z_{l k}\left(m^{+}\right)|0\rangle
\end{aligned}
$$

which are obtained by using (14) and (23). 


\section{Operator representation of the Dirac Coulomb Green function}

The use of the method of perturbation theory to solve the Dirac equation for a charged particle moving in the Coulomb field plus an electromagnetic field, which is considered as a perturbation with respect to the first field, always leads to equations like (10) and (11), where a perturbative term exists in the right-hand side of the equations. In order to solve these equations there is a need to establish the corresponding Green functions. In the traditional coordinate treatment, these Green functions are called the Dirac Coulomb Green functions (see Martin and Glauber 1958, Zapryagaev et al 1985, Gorshkov 1964, Zon et al $1972, \ldots$ ). In our description, (10) and (11) have a structure which is convenient for establishing the Green operator through the annihilation and creation operators and for transforming this operator into a normal form.

Let us consider, for example, (10) and (11) with an inhomogeneous term on the righthand side. In order to solve the above-mentioned equations we, first of all, have to exclude the existence of singularities appearing in places where $\xi_{s} \xi_{s}^{*} \rightarrow 0$ and expand the inhomogeneous term in power series of eigenvectors of the operators $\hat{L}^{2}$ and $\hat{\kappa}$. In this regard we represent wavefunctions in the form

$$
\Phi=\sum_{l, k}\left(\xi_{s} \xi_{s}^{*}\right)^{y} \tilde{\Phi}_{l k}
$$

where $\gamma=-l-1+\sqrt{\kappa^{2}-Z^{2} e^{4}}$. Thus, the above-mentioned equations lead to the following equations for the wavefunctions $\tilde{\Phi}_{l k}$ :

$$
\begin{gathered}
\left\{\left(\xi_{s} \frac{\partial}{\partial \xi_{s}}+\xi_{s}^{*} \frac{\partial}{\partial \xi_{s}^{*}}-1-l\right)^{2}+2(\gamma+l+1)\left(\xi_{s} \frac{\partial}{\partial \xi_{s}}+\xi_{s}^{*} \frac{\partial}{\partial \xi_{s}^{*}}-1-l\right)\right. \\
\left.-\left(\omega \xi_{s} \xi_{s}^{*}\right)^{2}+\left(2 Z e^{2} \varepsilon \pm \omega\right) \xi_{s} \xi_{s}^{*}\right\} \tilde{\Phi}_{l k}(\xi)=\tilde{\Phi}_{l k}^{(o)}(\xi) .
\end{gathered}
$$

The operator in (26) can be rewritten in the form

$$
\begin{array}{r}
\hat{H}_{ \pm}=\frac{1}{4}\left(M-M^{+}-2 l-2\right)^{2}+(\gamma+l+1)\left(M-M^{+}-2 l-2\right) \\
-\frac{1}{4}\left(M+M^{+}+N+2\right)^{2}+\alpha_{ \pm}\left(M+M^{+}+N+2\right)
\end{array}
$$

where $\alpha_{ \pm}=Z e^{2} \varepsilon / \omega \pm \frac{1}{2}$. Henceforth, for simplicity we leave out the signs \pm in the description. By using the Green function operator $\hat{G}_{l k}$ which satisfies the equation

$$
\hat{\boldsymbol{G}}_{l k} \hat{H} \tilde{\Phi}_{l k}(\xi)=\widetilde{\Phi}_{l k}(\xi)
$$

we establish the solution of (26) as follows:

$$
\widetilde{\Phi}_{l k}(\xi)=\hat{G}_{l k} \tilde{\Phi}_{l k}^{(o)}(\xi) .
$$

In order to establish $\hat{G}_{l k}$ we turn to (28) and rewrite it in the overt form

$$
\begin{gathered}
\hat{G}_{l \kappa}\left\{\frac{1}{4}\left(M-M^{+}-2 l-2\right)^{2}+(\gamma+l+1)\left(M-M^{+}-2 l-2\right)-\frac{1}{4}\left(M+M^{+}+N+2\right)^{2}\right. \\
\left.+\alpha_{ \pm}\left(M+M^{+}+N+2\right)\right\}\left(M^{+}\right)^{n} Z_{l k}\left(m^{+}\right)|0\rangle=\left(M^{+}\right)^{n} Z_{l k}\left(m^{+}\right)|0\rangle .
\end{gathered}
$$

By using correlations (25), equation (29) can be transformed into

$$
\begin{gathered}
\hat{G}_{l k}\left(M+M^{+}+N+2\right)\left\{-\left(n+\gamma+l+\frac{3}{2}-\alpha_{ \pm}\right)\left(M^{+}\right)^{n}-(2 \gamma+1)(-1)^{n} n !\right. \\
\left.\sum_{k=0}^{n-1} \frac{(-1)^{k}}{k !}\left(M^{+}\right)^{k}\right\} Z_{l k}\left(m^{+}\right)|0\rangle=\left(M^{+}\right)^{n} Z_{l k}\left(m^{+}\right)|0\rangle
\end{gathered}
$$


which suggests that the operator $\hat{G}_{l \kappa}$ should be found in the form

$$
\hat{G}_{l k}=\hat{B}_{l \kappa} \frac{1}{2+N+M+M^{+}} \quad \hat{B}_{l k}=\sum_{s=0}^{\infty} d_{s}(N / 2) M^{s}
$$

where $d_{s}(N / 2)$ are functions with argument $N / 2$. Further, we substitute (31) into (30), use correlations (25) in the action of operators and consider all the coefficients with the same vectors to be equal to zero. Consequently, we obtain the following system of algebraic equations for $d_{s}(N / 2)$ :

$$
\begin{aligned}
& -\left(n+\gamma+l+\frac{3}{2}-\alpha_{ \pm}\right) d_{0}(n+l)=1 \\
& (-1)^{s}\left(n+\gamma+l+\frac{3}{2}-\alpha_{ \pm}\right)(n+2 l+1) ! d_{s}(n-m+l) \\
& \quad+(2 \gamma+1) \sum_{t=0}^{s-1}(-1)^{t}(t+n-s+2 l+1) ! d_{t}(n-s+l)=0 \quad s=1,2, \ldots
\end{aligned}
$$

By solving the system of equations (32) with respect to unknown functions $d_{s}(n+l)$ we obtain

$$
\begin{aligned}
& d_{0}(n)=-\frac{1}{n+\gamma+\frac{3}{2}-\alpha_{ \pm}} \\
& d_{s}(n)=(-1)^{s}(2 \gamma+1) \frac{(n+l+1) !}{(n+s+l+1) !} \frac{\Gamma\left(n+s-\gamma+\frac{1}{2}-\alpha_{ \pm}\right) \Gamma\left(n+\gamma+\frac{3}{2}-\alpha_{ \pm}\right)}{\Gamma\left(n-\gamma+\frac{3}{2}-\alpha_{ \pm}\right) \Gamma\left(n+s+\gamma+\frac{5}{2}-\alpha_{ \pm}\right)} .
\end{aligned}
$$

As a result, the Green function operator is finally established in a normal form

$$
\begin{array}{r}
\hat{B}_{l \kappa}=\frac{1}{\frac{1}{2} N+\gamma+\frac{3}{2}-\alpha_{ \pm}}\left[-1+(2 \gamma+1) \sum_{s=1}^{\infty}(-1)^{s} \frac{\left(\frac{1}{2} N+l+1\right) !}{\left(\frac{1}{2} N+s+l+1\right) !}\right. \\
\left.\times \frac{\Gamma\left(\frac{1}{2} N+s-\gamma+\frac{1}{2}-\alpha_{ \pm}\right) \Gamma\left(\frac{1}{2} N+\gamma+\frac{5}{2}-\alpha_{ \pm}\right)}{\Gamma\left(\frac{1}{2} N-\gamma+\frac{3}{2}-\alpha_{ \pm}\right) \Gamma\left(\frac{1}{2} N+s+\gamma+\frac{5}{2}-\alpha_{ \pm}\right)} M^{s}\right] .
\end{array}
$$

The operator representation of the Green function (34) allows us in concrete calculations to reduce rather complicated calculations of matrix elements into purely algebraic operations like (25). In the next section we will demonstrate such algebraic calculations through the exemplary problem of relativistic hydrogen-like atoms in a homogeneous electric field.

\section{Relativistic polarizability of hydrogen-like atoms}

Let us now consider the Dirac equation for an electron with energy $\varepsilon_{\mathfrak{e}}$, moving in the Coulomb field of the nucleus with charge $Z e$ and in the external homogeneous electric field $E$. After transformation into $\xi$-space and after the substitution of (6) the Dirac equation leads to the following equations:

$$
\begin{aligned}
& \left(x_{\lambda} \frac{\partial}{\partial x_{\lambda}}+1+\omega_{\mathrm{e}} r-\frac{Z e^{2} \varepsilon_{\mathrm{e}}}{\omega_{\mathrm{e}}}\right) \tilde{F}-\left(\hat{\kappa}+\frac{Z e^{2}}{\omega_{\mathrm{e}}}\right) \widetilde{G}=-\frac{e E}{\omega_{\mathrm{e}}} r x_{3}\left(\varepsilon_{\mathrm{e}} \widetilde{F}+\widetilde{G}\right) \\
& \left(\hat{\kappa}-\frac{Z e^{2}}{\omega_{\mathrm{e}}}\right) \tilde{F}+\left(x_{\lambda} \frac{\partial}{\partial x_{\lambda}}+1-\omega_{\mathrm{e}} r+\frac{Z e^{2} \varepsilon_{\mathrm{e}}}{\omega_{\mathrm{e}}}\right) \tilde{G}=\frac{e E}{\omega_{\mathrm{e}}} r x_{3}\left(\tilde{F}+\varepsilon_{\mathrm{e}} \widetilde{G}\right)
\end{aligned}
$$


where $\omega_{\mathrm{e}}=\sqrt{1-\varepsilon_{\mathrm{e}}^{2}}$. Here and henceforth, all operators in (35) will be interpreted as operators in $\xi$-space corresponding to the formal changes

$$
\begin{aligned}
& r \rightarrow \xi_{s} \xi_{s}^{*} \quad x_{\lambda} \rightarrow\left(\tau_{\lambda}\right)_{s t} \xi_{s}^{*} \xi_{t} \\
& x_{\lambda} \frac{\partial}{\partial x_{\lambda}} \rightarrow \frac{1}{2}\left(\xi_{s} \frac{\partial}{\partial \xi_{s}}+\xi_{s}^{*} \frac{\partial}{\partial \xi_{s}^{*}}\right) \\
& \hat{l}_{\lambda} \rightarrow-\frac{1}{2}\left(\tau_{\lambda}\right)_{s t}\left(\xi_{t} \frac{\partial}{\partial \xi_{s}}-\xi_{s}^{*} \frac{\partial}{\partial \xi_{t}^{*}}\right) .
\end{aligned}
$$

Equations (35) will be solved using the method of perturbation theory with respect to the external electric field. Considering the dimensionless value of the strength of the electric field $E$ as a small parameter we expand the wavefunctions $\widetilde{F}$ and $\widetilde{G}$ in power series of this parameter

$$
\begin{aligned}
& \widetilde{F}=\widetilde{F}^{(0)}+E \widetilde{F}^{(1)}+E^{2} \widetilde{F}^{(2)}+\cdots \\
& \widetilde{G}=\widetilde{G}^{(0)}+E \widetilde{G}^{(1)}+E^{2} \widetilde{G}^{(2)}+\cdots
\end{aligned}
$$

By the expansion (37), $\varepsilon_{\mathrm{e}}$ and $\omega_{\mathrm{e}}$ for the ground state can be expressed as follows:

$$
\varepsilon_{\mathrm{e}}=\varepsilon-\frac{1}{2} a E^{2}+\cdots \quad \omega_{\mathrm{e}}=\omega+\frac{a \varepsilon}{2 \omega} E^{2}+\cdots
$$

where $a$ is defined as the polarizability of the hydrogen-like atoms. Here in (38), we take into account the well known fact that the linear Stark effect is absent for the ground state. By substituting (37) and (38) into (35), and considering to be zero all the coefficients with the same order of the parameter $E$, we obtain for every order of approximation the equations for wavefunctions $\widetilde{F}^{(k)}, \widetilde{G}^{(k)}$.

The formula for the ground state polarizability can thus be obtained

$$
a=-\frac{2 \omega e}{\varepsilon}\left(\left(\widetilde{F}^{(0)}\left|r x_{3}\right| \widetilde{F}^{(1)}\right\rangle-\varepsilon\left\{\tilde{F}^{(0)}\left|r x_{3}\right| \widetilde{G}^{(1)}\right\}\right) .
$$

The wavefunctions in the zero-order approximation $\widetilde{F}^{(0)}$ and $\widetilde{G}^{(0)}$ which, in fact, are solutions of equations like (10) and (11), can be established by using the algebraic method (see Komarov and Romanova 1985). We obtain

$$
\widetilde{F}^{(0)}=\frac{1}{(2 \omega)^{1-\varepsilon} \sqrt{\Gamma(2 \varepsilon)}} r^{\varepsilon-1}|0(\omega)\rangle \chi^{\dagger} \quad \widetilde{G}^{(0)}=0
$$

where $\omega=Z e^{2}, \varepsilon=\sqrt{1-Z^{2} e^{4}}$. Then, in equations for wavefunctions in the first-order approximation, the perturbation has the form

$$
\begin{aligned}
\frac{\operatorname{erx_{3}}}{\omega}\left(\varepsilon \widetilde{F}^{(0)}+\widetilde{G}^{(0)}\right) & =\frac{2 e \varepsilon}{(2 \omega)^{1-\varepsilon} \sqrt{\Gamma(2 \varepsilon)}} r^{\varepsilon} m_{3}^{+}|0(\omega)\rangle \chi^{\uparrow} \\
& =v_{\varepsilon} \varepsilon r^{\varepsilon}\left(\sqrt{2} Z_{1,2}+Z_{1,-1}\right)
\end{aligned}
$$

where

$$
v_{\varepsilon}=\frac{2 \sqrt{6} e(2 \omega)^{\varepsilon-3}}{3 \sqrt{\Gamma(2 \varepsilon)}} .
$$

It can easily be seen from (41) that $\widetilde{F}^{(1)}$ and $\widetilde{G}^{(1)}$ can be represented as follows:

$$
\widetilde{F}^{(1)}=\widetilde{F}_{+}^{(1)}+\widetilde{F}_{-}^{(1)} \quad \widetilde{G}^{(1)}=\widetilde{G}_{+}^{(1)}+\widetilde{G}_{-}^{(1)}
$$


where $\widetilde{F}_{ \pm}^{(1)}$ and $\widetilde{G}_{ \pm}^{(1)}$ are defined by the equations

$$
\begin{aligned}
& \left(x_{\lambda} \frac{\partial}{\partial x_{\lambda}}+1+\omega r-\frac{Z e^{2} \varepsilon}{\omega}\right) \widetilde{F}_{-}^{(1)}=-\varepsilon v_{\varepsilon} r^{\varepsilon} Z_{1,-1} \\
& \left(x_{\lambda} \frac{\partial}{\partial x_{\lambda}}+1-\omega r+\frac{Z e^{2} \varepsilon}{\omega}\right) \widetilde{G}_{-}^{(1)}-2 \widetilde{F}_{-}^{(1)}=-v_{\varepsilon} r^{\varepsilon} Z_{1,-1}
\end{aligned}
$$

and

$$
\begin{aligned}
& \left(x_{\lambda} \frac{\partial}{\partial x_{\lambda}}+1+\omega r-\frac{Z e^{2} \varepsilon}{\omega}\right) \widetilde{F}_{+}^{(1)}+3 \widetilde{G}_{+}^{(1)}=-\sqrt{2} \varepsilon v_{\varepsilon} r^{\varepsilon} Z_{1,2} \\
& \left(x_{\lambda} \frac{\partial}{\partial x_{\lambda}}+1-\omega r+\frac{Z e^{2} \varepsilon}{\omega}\right) \widetilde{G}_{+}^{(1)}+\widetilde{F}_{+}^{(1)}=-\sqrt{2} \varepsilon v_{\varepsilon} r^{\varepsilon} Z_{1,2} .
\end{aligned}
$$

In a simple way the solutions of (43) can be obtained in a form

$$
\begin{aligned}
& \widetilde{F}_{-}^{(1)}=\frac{1}{2} \varepsilon v_{\varepsilon} r^{\varepsilon-2}\left[\frac{2(\varepsilon+1)(2 \varepsilon+1)}{(2 \omega)^{3}}-r^{2}\right] Z_{1,-1} \\
& \widetilde{G}_{-}^{(1)}=-\frac{1+\varepsilon}{2 \omega} v_{\varepsilon} r^{\varepsilon-2}\left[r+\frac{2 \varepsilon+1}{(2 \omega)}\right] Z_{1,-1} .
\end{aligned}
$$

The system of equations (44) is more complicated in comparison with (43) and, therefore, for its solution the Green function should be used. As a result, we obtain

$$
\begin{aligned}
& \widetilde{F}_{+}^{(1)}=\left(M^{+}+\frac{1}{2} N+1-\varepsilon\right) \widetilde{G}_{+}^{(1)}-\sqrt{2} v_{\varepsilon} r^{\varepsilon} Z_{1,2} \\
& \widetilde{G}_{+}^{(1)}=\sqrt{2}(2-\varepsilon) v_{\varepsilon} r^{\gamma} \hat{G}_{12} r^{-\gamma+\varepsilon} Z_{1,2} .
\end{aligned}
$$

Substituting $\widetilde{F}^{(1)}$ and $\widetilde{G}^{(1)}$ into (39) and by using (25) as well as the correlation

$$
r^{n} Z_{l k}=\frac{(2 \omega)^{-n}}{\Gamma(-n)} \sum_{s=0}^{\infty} \frac{(-1)^{s}}{s !} \frac{\Gamma(s-n) \Gamma(2+2 l+n)}{\Gamma(s+2+2 l)}\left(M^{+}\right)^{s} Z_{l k}
$$

we finally obtain the formula for the polarizability

$$
a=a_{0}+\delta
$$

with

$$
\begin{gathered}
a_{0}=\frac{e^{2}(\varepsilon+1)(2 \varepsilon+1)\left(4 \varepsilon^{2}+13 \varepsilon+12\right)}{36 \omega^{4}}-\frac{e^{2}(\varepsilon-2)^{2} \Gamma(\varepsilon+\gamma+4) \Gamma(3+\varepsilon-\gamma)}{216(\gamma-\varepsilon+3) \Gamma(2 \varepsilon) \varepsilon \omega^{4}} \\
\delta=-\frac{e^{2}(\varepsilon-2)^{2} \Gamma(\varepsilon+\gamma+4) \Gamma(3+\varepsilon-\gamma)}{36 \varepsilon \omega^{4} \Gamma(2 \varepsilon) \Gamma(-\varepsilon-\gamma) \Gamma(-\varepsilon+\gamma+1)} \times\left(\sum_{k=1}^{\infty} \frac{\Gamma(k-\varepsilon-\gamma) \Gamma(k-\varepsilon+\gamma+1)}{k !(k+3) !(k+\gamma-\varepsilon+3)}\right. \\
-(2 \gamma+1) \sum_{q=1}^{\infty} \frac{\Gamma(q-\varepsilon+\gamma+1) \Gamma(q-\varepsilon-\gamma+2)}{(q+3) ! \Gamma(q-\varepsilon+\gamma+4)} \\
\left.\times \sum_{s=0}^{q-1} \frac{\Gamma(s-\varepsilon-\gamma) \Gamma(s-\varepsilon+\gamma+3)}{s ! \Gamma(s-\varepsilon-\gamma+3)}\right)
\end{gathered}
$$

where $\gamma=-2+\sqrt{4-Z^{2} e^{4}}$.

The numerical values of $a$ and $a_{0}$ for $Z$ ranging from 1 to 137 are given in table 1 . The corresponding graph describing the dependence of the polarizability on nuclear charge $Z$ is also given (see figure 1). Taking into account the fact that $Z e^{2}<1$ for $Z \leqslant 137$ we expand $a$ in a power series of $Z e^{2}$

$$
a=\frac{9 e^{2}}{2\left(Z e^{2}\right)^{4}}\left[1-\frac{28}{27}\left(Z e^{2}\right)^{2}+\frac{2 \pi^{2}+31}{432}\left(Z e^{2}\right)^{4}+\cdots\right] .
$$


$\mathrm{rel} /$ nonrel

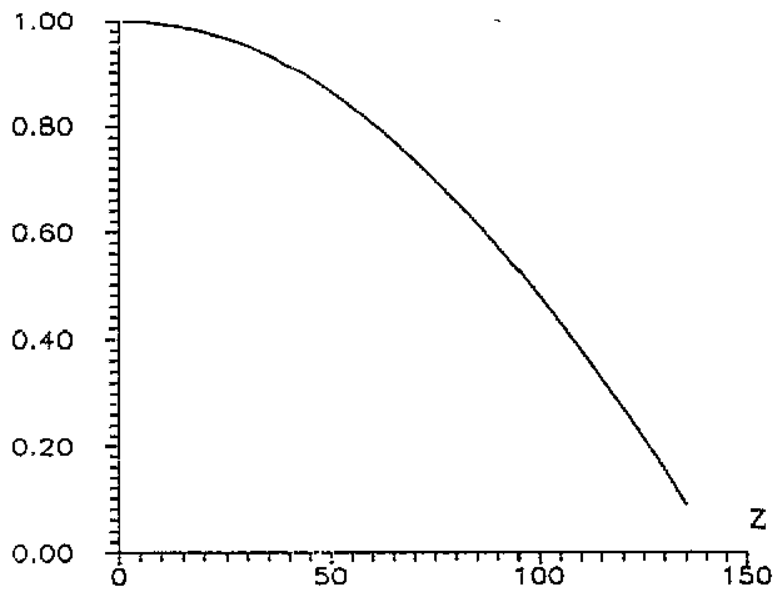

Figure 1. The dependence of the polarizability on nuclear charge $Z$.

Table 1. Relativistic polarizability in the ground state of hydrogen-like atoms; $a, a_{\text {non }}$ are the relativistic and non-relativistic polarizabilities respectively; $a_{\text {non }}=9 e^{2} / 2\left(Z e^{2}\right)^{-4} ; a_{1}$ is an analytical estimation term of $a$.

\begin{tabular}{rlllll}
\hline$z$ & $a / a_{\text {non }}$ & $a_{0} / a_{\text {non }}$ & \multicolumn{1}{l}{$z$} & $a / a_{\text {non }}$ & $a_{0} / a_{\text {non }}$ \\
\hline 1 & 0.999945 & 0.999945 & 40 & 0.912499 & 0.912099 \\
2 & 0.999779 & 0.999778 & 45 & 0.889547 & 0.889039 \\
3 & 0.999503 & 0.999501 & 50 & 0.864040 & 0.863412 \\
4 & 0.999117 & 0.999113 & 55 & 0.836027 & 0.835265 \\
5 & 0.998620 & 0.998613 & 60 & 0.805562 & 0.804655 \\
6 & 0.998012 & 0.998004 & 65 & 0.772707 & 0.771642 \\
7 & 0.997295 & 0.997283 & 70 & 0.737526 & 0.736294 \\
8 & 0.996467 & 0.996451 & 75 & 0.700091 & 0.698683 \\
9 & 0.995529 & 0.995509 & 80 & 0.660478 & 0.658887 \\
10 & 0.994481 & 0.994456 & 85 & 0.618769 & 0.616993 \\
11 & 0.993323 & 0.993292 & 90 & 0.575049 & 0.573088 \\
12 & 0.992055 & 0.992019 & 95 & 0.529404 & 0.527267 \\
13 & 0.990677 & 0.990635 & 100 & 0.481922 & 0.479623 \\
14 & 0.989189 & 0.989141 & 105 & 0.432680 & 0.430250 \\
15 & 0.987592 & 0.987536 & 110 & 0.381741 & 0.379222 \\
16 & 0.985885 & 0.985821 & 115 & 0.329122 & 0.326582 \\
17 & 0.984068 & 0.983997 & 120 & 0.274744 & 0.272283 \\
18 & 0.982143 & 0.982062 & 125 & 0.218279 & 0.216049 \\
19 & 0.980108 & 0.980018 & 130 & 0.158625 & 0.156872 \\
20 & 0.977964 & 0.977865 & 135 & 0.090429 & 0.089657 \\
25 & 0.965616 & 0.965461 & 136 & 0.073004 & 0.072582 \\
30 & 0.950569 & 0.950345 & 137 & 0.045002 & 0.045236 \\
35 & 0.932853 & 0.932547 & & & \\
\hline & & & & & \\
\hline
\end{tabular}

The numerical data of $a$ (see table 1) and the expansion (49) coincide with results given in Zon et al (1972). But in our results, it should be noted that the series appearing in $\delta$ are quickly convergent and the value $\delta / a_{0}$ for all values of $Z$ is very small. The biggest value, whereby $Z=130$, is $\delta / a_{0} \sim 0.01$. This result allows us to use $a_{0}$ as a simple analytical formula for the polarizability of the ground state of hydrogen-like atoms. In fact, it is useful for analysis of different effects connected with the problem of interaction of two 
hydrogen-like atoms having high values of nuclear charge (see Baras 1988). Furthermore, we consider it interesting and useful to scale the polarizability with various size parameters. The size of hydrogen-like atom could be defined as follows:

$$
\tilde{r}=\int \mathrm{d}^{3} r|\Psi(r)|^{2} r
$$

For the ground state of hydrogen-like atoms, we have

$$
\tilde{r}=\frac{\left\langle\widetilde{F}^{(0)}\left|r^{2}\right| \tilde{F}^{(0)}\right\rangle}{\left\langle\widetilde{F}^{(0)}|r| \tilde{F}^{(0)}\right\rangle}=\frac{2 \sqrt{1-Z^{2} e^{4}}+1}{2 Z e^{2}}
$$

from which it follows that

$$
\tilde{r}^{4} \simeq 5.06\left(1-\frac{4}{3}\left(Z e^{2}\right)^{2}+\frac{1}{3}\left(Z e^{2}\right)^{4}+\cdots\right) \frac{1}{\left(Z e^{2}\right)^{4}} .
$$

The comparison of (49) with (50) leads to the conclusion that the relativistic polarizability scales like $\tilde{r}^{4}$ for low values of nuclear charge.

It should be noted that the high convergency of the power series appearing in the expression for $\delta$ as well as the high accuracy of the analytical estimation $a_{0}$ are directly related to the expansion (34) of the Dirac Coulomb Green function. This expansion in the coordinate representation leads to power series of the oscillator wavefunctions. The results obtained serve as a good example for demonstrating the advantage of the latter in comparison with the use of the usual representations which lead to power series of the Coulomb wavefunctions. In our next work, we will show the above-mentioned efficiency in calculations of the dynamical relativistic polarizability of hydrogen-like atoms, a problem which is rarely investigated because of its complexity.

\section{Acknowledgments}

One of the authors (LVH) would like to thank Dr A V Yukhnhevich (Institute of PhysicoChemical Problems, Belarussian State University) for many useful discussions about the application of the algebraic approach to the problem of hydrogen-like atoms on silicon single-crystal surfaces, which has strongly stimulated this present research.

This work was supported in part by the Fundamental Research Foundation of the Republic of Belarus.

\section{References}

Baras Yu S 1988 Van der Waals Forces (Moscow: Nauka)

Bartlett M L and Power E A 1969 J. Phys. A: Math. Gen. 2419

Barut A O and Nagel J 1976 Phys. Rev. D 132075

Gorshkov V G 1964 Zh. Eksp. Teor. Fiz. 471984

Kibler M and Negadi T 1983 J, Phys. A: Math. Gen. 164265

Komarov L I and Romanova T S 1982 lzv. Akad. Nauk BSSR (Ser. Fis.-Mat. Nauk) 298

1985 J. Phys. B: At. Mol. Phys. 18859

Labzowsky L N 1973 Vestnik Leningrad State University 10 no 219

Le Van Hoang, Komarov L I and Romanova T S 1989 J. Phys. A: Math. Gen. 221543

Le Van Hoang, Ly Xuan Hai, Komarov L I and Romanova T S 1992 J. Phys. A: Math. Gen. 256461

Manakov N L, Rapoport L P and Zapryagaev S A 1973 Phys. Lett. 43A 139

Martin P C and Glauber R J 1958 Phys. Rev. A 1091307

McDowell H K and Porter R N 1977 J. Chem. Phys. 664725

Swainson R A and Dracke G W F 1991 J. Phys. A: Math. Gen. 2495 
Zapryagney S A and Manakov N L 1981 lzw. Akad. Nauk USSR (Ser. Phys.) 452336

Zapryageev S A, Manakov N L and Palchikov V G 1985 Theory of Multiply Charged lons with One and Two Electrons (Moscow: Energontomizdat)

Zon B A, Manakov N L and Rapoport L P 1972 Yad. Fiz. 15508 